\title{
RADIÓLISE DE AMINOÁCIDO SOB AÇÃO DE ÍONS PESADOS: IMPLICAÇÕES EM ASTROQUÍMICA E ASTROBIOLOGIA
}

\section{RADIOLYSIS OF AMINOACID UNDER ACTION OF HEAVY IONS: IMPLICATIONS IN ASTROCHEMISTRY AND ASTROBIOLOGY}

\author{
Williamary Portugal ${ }^{1}$ \\ Sergio Pilling ${ }^{2}$ \\ Diana P. P. Andrade ${ }^{2}$ \\ Philippe Boduch ${ }^{3}$ \\ Hermann Rothard ${ }^{3}$
}

RESUMO: Neste trabalho, estudamos a estabilidade da molécula de glicina na sua forma zwiteriônica cristalina, conhecida como $\alpha$-glycina ( ${ }^{+} \mathrm{NH}_{3} \mathrm{CH}_{2} \mathrm{COO}$ ) a duas temperaturas (14 e $300 \mathrm{~K}$ ), sob a ação de íons rápidos, simulando ambientes espaciais na presença de raios cósmicos pesados. Para tanto, a meia vida da glicina bombardeada no meio interestelar (nuvens densas) foi estimada, sendo $1.1 \times 10^{4}$ anos (300 K) e $1.6 \times 10^{3}$ anos (14 K). No sistema solar, os valores foram de $1.2 \times 10^{3}$ anos (300 K) e $2.8 \times 10^{3}$ anos (14 K). O estudo da estabilidade da glicina, nesses ambientes, traz à tona questões sobre o papel dessa molécula na química prébiótica na Terra, para auxiliar nas descobertas sobre a origem da vida como a conhecemos.

Palavras-chave: astrobiologia; química pré-biótica; resistência à radiação; moléculas interestelares; glicina.

ABSTRACT: This work studied the stability of the glycine molecule in the crystalline zwitterion form, known as $\alpha$ glycine ( ${ }^{+} \mathrm{NH}_{3} \mathrm{CH}_{2} \mathrm{COO}$ ) at two temperatures (14 and $300 \mathrm{~K}$ ), under action of heavy ions simulating special environments in the presence of the hard cosmic rays. The half-live of glycine in interstellar medium was estimated to be $1.1 \times 10^{4}$ years $(300 \mathrm{~K})$ and $1.6 \times 10^{3}$ years $(14 \mathrm{~K})$. In the Solar System, the values were $2.9 \times 10^{4}$ years $(300 \mathrm{~K})$ and $1.6 \times 10^{4}$ years $(14 \mathrm{~K})$. The study of the stability the glycine in these environments raises questions about the role of this specie in the pre-biotic chemistry to help discover the origin of life, as we know it. Keywords: astrobiology; prebiotic chemistry; radiation resistance interstellar molecules; glycine.

\footnotetext{
1 Doutoranda em Física e Astronomia na Universidade do Vale do Paraíba - Univap / IP\&D. E-mail: williamary@uol.com.br.

2 Docente da Univap / IP\&D - Laboratório de Física e Astronomia. E-mail: sergiopilling@yahoo.com.br.

${ }^{3}$ Centre de Recherche sur les lons, les Matériaux et la Photonique CIMAP GANIL/CEA/CNRS/ENSICAEN/Université de Caen Basse-Normandie), França. E-mail: boduch@ganil.fr. 


\section{INTRODUÇÃO}

Densas regiões do Meio Interestelar (MI), chamadas nuvens moleculares, são caracterizadas por temperaturas muito baixas, na ordem de $10-30 \mathrm{~K}$ e densidade em torno de $10^{4}-10^{8}$ partículas $\mathrm{cm}^{-3}$. Devido à baixa temperatura, moléculas na fase gasosa são adsorvidas na superfície dos grãos de poeira, produzindo um manto rico de gelo com água e uma variedade de moléculas simples, como $\mathrm{CO}_{2}, \mathrm{CO}, \mathrm{CH}_{3} \mathrm{OH}$ e $\mathrm{NH}_{3}$ (BOOGERT; EHRENFREUND, 2004).

Estudos em laboratórios têm mostrado que a fotólise e a radiólise de gelos análogos aos do espaço por partículas de alta energia vindas do sistema solar (SS) (ex.: raios cósmicos, radiação UV, raios $\mathrm{X}$, elétrons e prótons) (SHEN et al., 2004), podem produzir compostos orgânicos complexos e moléculas pré-bióticas, tais como aminoácidos e nucleobases (MUÑOZ et al., 2002; KOBAYASHI et al., 2008; PILLING et al., 2009). A presença desses compostos pré-bióticos foi, também, observada em análises de laboratório de amostras de meteoritos, onde mais de 70 aminoácidos (dentre eles a glicina) foram identificados, como no caso do meteorito Murchison (CRONIN; PIZZARELLO, 1983; GLAVIN; DWORKIN, 2009; GLAVIN et al., 2011). O entendimento sobre 0 comportamento da interação entre essas partículas de alta energia com as diversas superfícies espaciais (ex.: gelos interestelares, cometas, luas, meteoritos, asteroides) auxilia a compreensão do seu papel na evolução da química interestelar e pode trazer à tona informações sobre os primeiros passos para a origem da vida.

\section{METODOLOGIA}

Os experimentos ocorreram em uma câmara de aço inoxidável, sob condições de ultra-alto vácuo acoplada à linha experimental IRRSUD (IRRadiation SUD) do acelerador de íons pesados GANIL (Grand Accélérateur National d'lons Lourds), em Caen, França. As amostras de cristais de $\alpha$-glicina foram preparadas fora da câmara de vácuo, por gotejamento da solução aquosa da molécula ( 0.1 Molar) em um substrato de Csl. Os experimentos foram realizados em duas diferentes temperaturas: 14 e $300 \mathrm{~K}$ para simular ambientes, tais como grãos de poeira em nuvens moleculares densas, discos protoplanetários e cometas. A primeira amostra foi inserida na câmara e bombardeada a 300K. A segunda, foi inserida a $300 \mathrm{~K}$ e resfriada até $14 \mathrm{~K}$, temperatura na qual ocorreu a segunda análise da amostra. As amostras foram irradiadas por íons de ${ }^{58} \mathrm{Ni}^{11+}$, com energia de $46 \mathrm{MeV}$ e fluxo de $2 \times 10^{9}$ íons $\mathrm{cm}^{-2} \mathrm{~s}^{-1}$, até a fluência de $1 \times 10^{13}$ íons $\mathrm{cm}^{-2}$.

Os espectros de infravermelho da glicina antes e depois de diferentes fluências foram obtidos in-situ pelo espectrômetro infravermelho de Transformada de Fourrier (FTIR) um Nicolet - Magma 550, com intervalo de número de onda de 4000 até $650 \mathrm{~cm}^{-1}$ e resolução de $1 \mathrm{~cm}^{-1}$.

\section{RESULTADOS}

Os espectros IR, obtidos das amostras de $\alpha$-glicina não irradiadas e irradiadas em diferentes fluências, são apresentados nas Figuras $1 \mathrm{a}$ e $1 \mathrm{~b}$, respectivamente, para a amostra a 14 e $300 \mathrm{~K}$. Em ambas as Figuras, a amostra cristalina não irradiada é 
representada pelo espectro superior. A seta próxima do pico em $1034 \mathrm{~cm}^{-1}$ indica o modo vibracional de estiramento da ligação $\mathrm{C}-\mathrm{N}$ na molécula de glicina, empregado para quantificar e estudar a estabilidade da amostra.
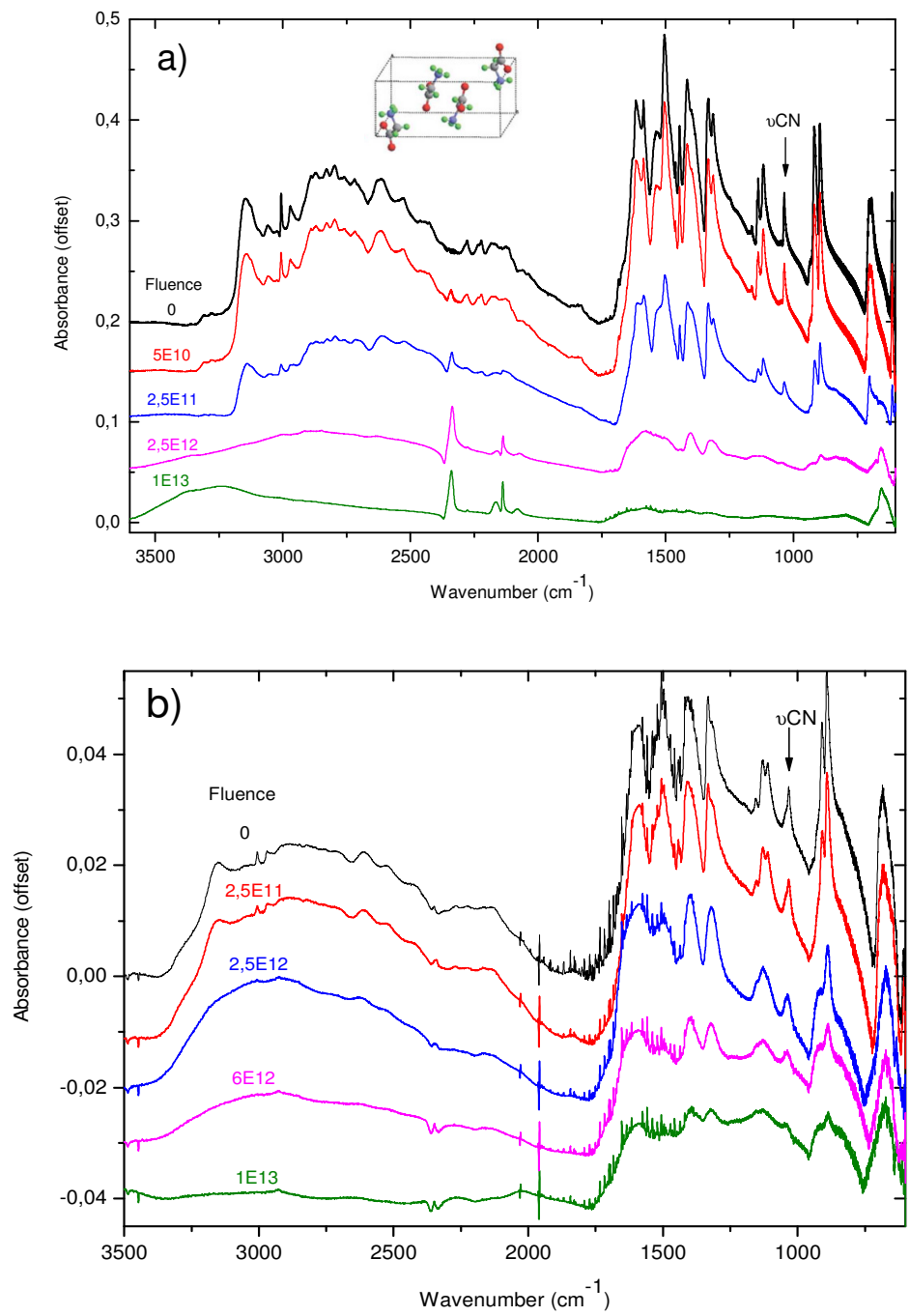

Figura 1 - Espectro infravermelho da $\alpha$-glicina $\left({ }^{+} \mathrm{NH}_{3} \mathrm{CH}_{2} \mathrm{COO}-\right)$ durante os experimentos em diferentes fluências de íons. a) amostra a $14 \mathrm{~K}$; b) amostra a $300 \mathrm{~K}$.

As seções de choque de dissociação da glicina a 14 e a $300 \mathrm{~K}$, sob ação dos íons de $\mathrm{Ni}^{11+}$, foram obtidas pela expressão

$$
\ln \left(\frac{a}{a_{0}}\right)=\sigma_{d} \cdot F
$$

onde $a_{0}$ e a são a absorbância integrada de um dado modo vibracional no início do experimento e a uma dada fluência, respectivamente. A $\sigma_{d}$ é a seção de choque de destruição em $\mathrm{cm}^{2}$ e $\mathrm{F}$ é a fluência em íons $\mathrm{cm}^{-2}$. Os valores, considerando a ligação $\mathrm{C}-\mathrm{N}$, foram estimados em $3.0 \times 10^{-12}$ $\mathrm{cm}^{2}$ e $1.3 \times 10^{-13}$, para as amostras a 14 e a $300 \mathrm{~K}$, respectivamente. A partir das seções de choque de destruição $\left(\sigma_{d}\right)$, determinadas neste trabalho, foi possível calcular as seções de choque de destruição da 
molécula de glicina sob ação de todos os outros íons constituintes dos raios cósmicos em função da energia, representados pelos elementos da tabela periódica de maior abundância nos raios cósmicos $(12 \leq Z \leq 29$ além de $\mathrm{H}$, He, $\mathrm{O}$ e $\mathrm{C}$ ). Para tanto, utilizamos a expressão [3], onde as seções de choque dependem do $S_{e}$ (stopping power eletrônico).

$$
\sigma_{d}=a S e^{n}
$$

$\mathrm{Na}$ expressão, a e $n$ são valores encontrados pelo ajuste de curva, como mostrado na Figura 2.

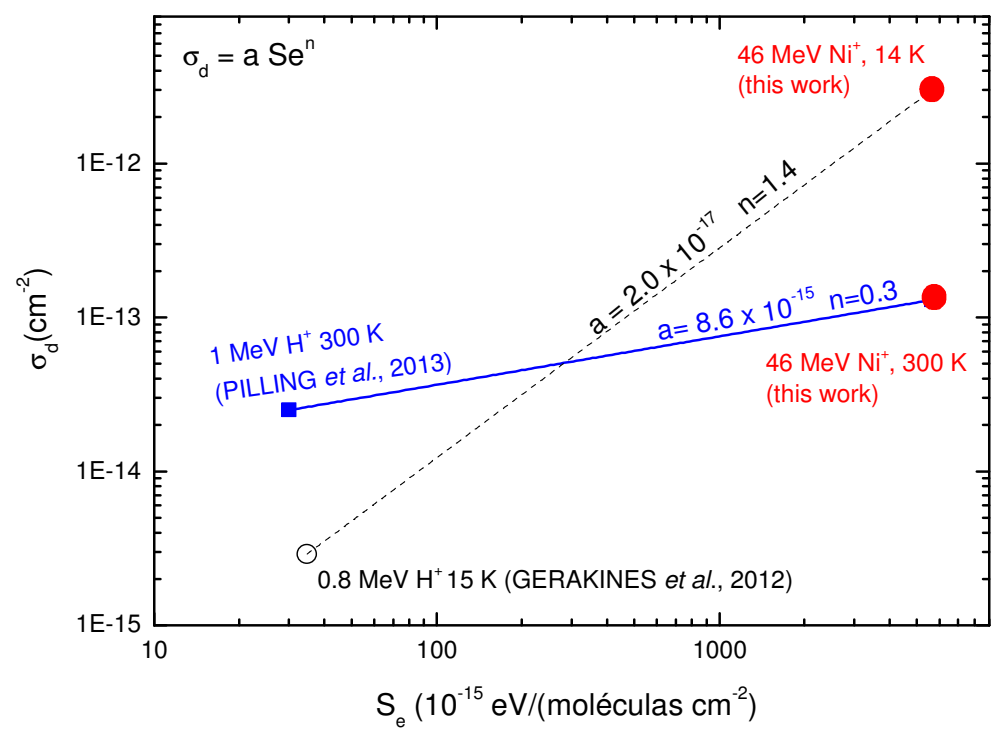

Figura 2 - Dependência da seção de choque de destruição da glicina em relação ao stopping power eletrônico nas duas temperaturas ( 14 e $300 \mathrm{~K}$ ). Os círculos vermelhos representam os dados referentes ao íon ${ }^{58} \mathrm{Ni}^{11+}$ a 14 e $300 \mathrm{~K}$ utilizado nesse trabalho.

Para estimar a meia-vida $\left(\tau_{1 / 2}\right)$ de cristais de $\alpha$-glicina no MI e SS, pelo método de Andrade et al. (2013), utilizamos a equação

$$
\tau=\frac{\ln (2)}{k}[s]
$$

onde k (taxa de dissociação) é a soma $\sum\left(\int \Phi_{Z}(\mathrm{E}) \sigma_{d, Z}(\mathrm{E}) d \mathrm{E}\right)$. Nessa soma, $\Phi_{z}$ é a densidade do fluxo estimado de raios cósmicos $(\Phi H C R(E))$ entre $E$ e $E+d E$ em íons $\mathrm{cm}^{-2} \mathrm{~s}^{-1}(\mathrm{MeV} / \mathrm{u})^{-1}$ e $\sigma_{d, z}$ a seção de choque de destruição em $\mathrm{cm}^{2}$, em função da energia para um dado íon $z$. A integração foi realizada na faixa total de energia estudada $0.1-1.5 \times 10^{3} \mathrm{MeV} / \mathrm{u}$, sobre a área abaixo de uma dada curva dos gráficos referentes à taxa de dissociação da glicina em função da energia no MI e SS. Maiores detalhes podem ser encontrados em Portugal (2013).

Finalmente, as meias vidas da molécula de glicina $(\tau)$ no $\mathrm{Ml}$ e SS, a 14 e $300 \mathrm{~K}$ puderam ser estimadas para 0 alcance de energia de $0.1-1.5 \times 10^{3}$ $\mathrm{MeV} / \mathrm{u}$. No MI, os valores de tempo de meia vida foram de $1.1 \times 10^{4}$ anos $(300 \mathrm{~K})$ e $1.6 \mathrm{x}$ $10^{3}$ anos (14 K). No SS, esses valores foram de $1.2 \times 10^{3}$ anos $(300 \mathrm{~K})$ e $2.8 \times 10^{3}$ anos $(14 \mathrm{~K})$. Outros valores de meia vida foram estimados, levando em consideração o intervalo de energia de $\sim 0.1-10 \mathrm{MeV} / \mathrm{u}$ e a ação apenas dos íons pesados $(12 \leq Z \leq 29)$ (ver Tabela 1). 
Tabela 1 - Meias vidas $\tau$ (em anos) da molécula da glicina no MI e SS, a 14 e $300 \mathrm{~K}$ exposta à irradiação dos raios cósmicos

\begin{tabular}{ccccc} 
& \multicolumn{2}{c}{ MI (nuvens densas) } & \multicolumn{2}{c}{ SS } \\
\hline & $\mathbf{1 4 K}$ & $\mathbf{3 0 0 K}$ & $\mathbf{1 4 K}$ & $\mathbf{3 0 0 K}$ \\
\hline$\tau$ & $1.6 \times 10^{3}$ & $1.1 \times 10^{4}$ & $2.8 \times 10^{3}$ & $1.2 \times 10^{3}$ \\
\hline$\tau^{\prime}$ & $1.8 \times 10^{3}$ & $2.7 \times 10^{4}$ & $2.8 \times 10^{3}$ & $1.2 \times 10^{3}$ \\
$\tau_{\text {HCR }}$ & $\sim 1.6 \times 10^{3}$ & $2.6 \times 10^{4}$ & $2.9 \times 10^{5}$ & $3.8 \times 10^{6}$ \\
$\tau_{\text {HCR }}$ & $1.8 \times 10^{3}$ & $3.7 \times 10^{4}$ & $2.9 \times 10^{5}$ & $3.8 \times 10^{6}$ \\
$\tau / \tau_{\text {HCR }}$ & $\sim 1.0$ & 0.42 & $9.6 \times 10^{-3}$ & $3.2 \times 10^{-4}$ \\
\hline
\end{tabular}

$\tau$ : Meia vida da glicina devido à interação de todos os íons (12 $\leq \mathrm{Z} \leq 29, \mathrm{H}, \mathrm{He}, \mathrm{C} \mathrm{e} \mathrm{O}$ ) no intervalo de energia de $\sim 0.1-1.5 \times 10^{3} \mathrm{MeV} / \mathrm{u}$. $\tau^{\prime}$ : Meia vida da glicina devido à interação de todos os íons $(12 \leq \mathrm{Z} \leq 29, \mathrm{H}$, $\mathrm{He}, \mathrm{C} \mathrm{e} \mathrm{O)} \mathrm{no} \mathrm{intervalo} \mathrm{de} \mathrm{energia} \mathrm{de} \sim 0.1-10 \mathrm{MeV} / \mathrm{u}$. $\tau_{\mathrm{HCR}}$ : Meia vida da glicina devido à interação dos íons pesados $(12 \leq \mathrm{Z} \leq 29)$ no intervalo de energia de $\sim 0.1-1.5 \times 10^{3} \mathrm{MeV} / \mathrm{u}$. $\tau_{\mathrm{HCR}}$ : Meia vida da glicina devido à interação dos íons pesados $(12 \leq Z \leq 29)$ no intervalo de energia de $\sim 0.1-10 \mathrm{MeV} / \mathrm{u}$.

\section{CONCLUSÕES}

Nossos principais resultados e conclusões foram os seguintes: as meias vidas da glicina, no MI, foram estimadas utilizando a metodologia descrita em Andrade et al. (2013), sendo: $1.1 \times 10^{4}$ anos (para a amostra a $300 \mathrm{~K}$ ) e $1.6 \times 10^{3}$ anos $(14 \mathrm{~K})$. No SS, os valores foram $1.2 \times 10^{3}$ anos $(300 \mathrm{~K})$ e $2.8 \times 10^{3}$ anos $(14 \mathrm{~K})$. Podese observar que, no Ml, a amostra estudada em temperatura mais alta tem meia vida maior. Já, no SS, em ambientes mais quentes e sob a ação de todos os íons constituintes dos raios cósmicos (incluindo os íons pesados $(12 \leq Z \leq 29)$ e os íons de $H$, $\mathrm{He}, \mathrm{O}$ e $\mathrm{C}$ ), a meia vida da molécula é menor. Isso se deve ao fato de que no $\mathrm{MI}$ prevalece o efeito dos íons pesados sobre o conjunto total dos íons constituintes dos raios cósmicos, sendo a taxa de dissociação da glicina maior a 14 do que a $300 \mathrm{~K}$. No SS, o efeito dos íons leves, especificamente do $\mathrm{H}$ e do $\mathrm{He}$, oriundos principalmente do sol, torna-se importante. Além disso, a seção de choque da glicina referente à ação do $\mathrm{H}$ e do He é maior em ambientes mais quentes do SS.

Com esses resultados, pode-se concluir que, para que a glicina tenha sobrevivido durante a formação do SS sob o bombardeamento constante dos raios cósmicos, a molécula deveria encontrar-se protegida por camadas de outros compostos ou de minerais, como, por exemplo, abaixo de cerca de $20 \mu \mathrm{m}$ de material (no caso do bombardeio por íons de $\mathrm{Ni}$ a $46 \mathrm{MeV}$ ). Isso poderia ocorrer com moléculas presentes no interior de cometas, asteroides ou, mesmo, de grãos interestelares de grandes dimensões. Essas moléculas protegidas da radiação teriam um tempo de meia vida maior do que aquelas diretamente expostas ao campo de radiação, possibilitando sua participação na química pré-biótica que culminou com a origem da vida em nosso planeta.

\section{REFERÊNCIAS}

ANDRADE, D. P. P. et al. Chemical 
Reactions induced in frozen formic acid by in dense clouds and their relevance to origins heavy ion cosmic rays. Mon. Not. R. Astron. of bio-homochirality. Proc. IAU Symp., v. 251, Soc., v. 430, p. 787-796, 2013.

p. 465, 2008.

BOOGERT, A. C. A.; EHRENFREUND, P. Interstellar Ices. Astrophysics of Dust. ASP Conference Series, v. 309, p. 547, 2004.

MUÑOZ CARO, G. M. et al. Amino acids from ultraviolet irradiation of interstellar ice analogues. Nature, v. 416, p. 403-406, 2002.

CRONIN, J. R.; PIZZARELlO, S. Amino PILLING, S. et al. DNA Nucleobase acids in meteorites. Adv. Space Res. v. 3, n, Synthesis at Titan Atmosphere Analog by 9, p. 5-18, 1983. Soft X-rays. J. Phys. Chem. A. v. 113, p. GLAVIN, D. P.; DWORKIN, J. P. Enrichment of the amino acid L-isovaline by aqueous alteration on $\mathrm{Cl}$ and $\mathrm{CM}$ meteorite parent bodies. Proc. Natl. Acad. Sci. USA, v. 106, p.5487-5492, 2009.

GLAVIN, D. P. et al. The effects of parent body processes on amino acids in carbonaceous chondrites. Meteoritics and Planetary Science, v. 45, p.1948-1972, 2011.

KOBAYASHI, K. et al. Formation of amino acid precursors with large molecular weight

PORTUGAL, W. Radiólise da molécula de glicina empregando ions pesados em ambientes astrofísicos simulados: implicações em astroquímica e astrobiologia. 2013. Dissertação (Mestrado) Universidade do Vale do Paraíba, São José dos Campos, 2013.

SHEN et al. 2004. Cosmic ray induced explosive chemical desorption in dense clouds. Astron. astrophys., v. 415, p. 203215, 2004. 